Preprint PUPT-1681, DAMTP HEP/97-17

e-Print Archive: hep-ph/9703266

\title{
Lattice Chern-Simons Number Without Ultraviolet Problems
}

\author{
Guy D. Moore巾 \\ Princeton University \\ Joseph Henry Laboratories, PO Box 708 \\ Princeton, NJ 08544, USA \\ Neil Turok ? \\ DAMTP, Silver Street \\ Cambridge, CB3 $9 E W, U K$
}

\begin{abstract}
We develop a topological method of measuring Chern-Simons number change in the real time evolution of classical lattice $\mathrm{SU}(2)$ and $\mathrm{SU}(2)$ Higgs theory. We find that the ChernSimons number diffusion rate per physical 4-volume is very heavily suppressed in the broken phase, and that it decreases with lattice spacing in pure Yang-Mills theory, although not as quickly as predicted by Arnold, Son, and Yaffe.
\end{abstract}

\section{Introduction}

In pioneering work Andrei Sakharov [1] pointed out that unified theories of elementary particle physics had the potential to explain one of cosmology's basic mysteries, namely the overabundance of matter over antimatter in the universe. Since then, two main embodiments of this idea have emerged. Early work on the subject attempted to exploit the baryon number violation present in grand unified theories (GUTs), and this is still a popular approach. The idea here was simple, that particles whose decays violated baryon number might fall out of equilibrium as the universe cooled, and their decays could lead to a baryon asymmetry. However, as usual with GUTs the main problem is an overabundance of models and associated free parameters, which are difficult to experimentally constrain since most of the parameters are relevant to physics at inaccessibly high energies. Furthermore, the failure of experiments to detect evidence for GUT baryon number violation makes the prospect of experimental test seem at present remote.

The last decade has seen much more interest in the idea that much lower energy physics, within reach of particle accelerators and other experiments, might yet realize Sakharov's vision. It has been known since the work of t'Hooft that baryon number is violated in the Minimal Standard Model [2], for deep reasons relating to a) its chirality and b) the topology of the vacuum. The baryon number current $J_{\mu}^{B}$ is not conserved because only the left handed

\footnotetext{
${ }^{1}$ e-mail: guymoore@puhep1.princeton.edu

2e-mail: N.G.Turok@damtp.cam.ac.uk
} 
fields couple to the $\mathrm{SU}(2)$ gauge fields, and their fermion number current possesses an axial anomaly:

$$
\partial^{\mu} J_{\mu}^{B}=\frac{-N_{F} g^{2}}{32 \pi^{2}} \operatorname{Tr} \tilde{F}^{\mu \nu} F_{\mu \nu}=\frac{-N_{F} g^{2}}{32 \pi^{2}} \partial^{\mu} K_{\mu} .
$$

The charge associated with this anomaly, $\left(g^{2} / 32 \pi^{2}\right) \int d^{3} x K_{0} \equiv N_{C S}$, the Chern-Simons number, can take on any integer value in the vacuum because the gauge group $\mathrm{SU}(2)$ has a nontrivial third homotopy group. (Note that we have ignored the hypercharge term in equation (1), because its Chern-Simons number is forced to be zero in the vacuum, so it can never contribute to a 'permanent' change in the baryon number).

At first the violation of baryon number in the standard model was considered an irrelevant peculiarity, because at low energies, changes to $N_{C S}$ only occur due to quantum mechanical tunneling, which is exponentially suppressed by $O\left(\exp \left(-4 \pi / \alpha_{W}\right)\right) \sim \exp (-400)$. However, the rate need not be so suppressed at higher temperatures [3]. More careful consideration shows that transitions between vacua of differing $N_{C S}$ can be excited thermally at a rate which in the broken electroweak phase is dominated by trajectories which pass near to the minimal energy "Sphaleron" saddlepoint [四], and the exponential suppression is $\sim \exp \left(-E_{s p h} / T\right)=\exp \left(-2 B M_{W}(T) / \alpha_{W} T\right)$, with $M_{W}(T)=g_{W} v(T) / 2, v(T)$ the size of the Higgs condensate at temperature $T$, and $B$ a constant ranging from 1.5 to 2.7 as the zero temperature Higgs mass ranges from zero to infinity [5].

However, this semiclassical calculation breaks down in the symmetric electroweak phase. Here the Higgs condensate has dissolved and there is no barrier to thermally activated $N_{C S}$ change, and hence to baryon number violation. Infrared gauge field configurations are expected to dominate the diffusive behavior of $\int d^{4} x \tilde{F}^{\mu \nu} F_{\mu \nu}$, because the energy of intermediate field configurations which the system must pass through to permanently change $N_{C S}$, carrying half-integral $N_{C S}$, scales inversely with their size. Thus short wavelength (UV) contributions to $\tilde{F}^{\mu \nu} F_{\mu \nu}$ are expected to behave oscillatorily and not permanently change $N_{C S}$. In this case the diffusion of $N_{C S}$ is believed to be essentially classical since it involves high occupation number infrared fields, which should conform closely to the classical field approximation. In the classical field theory at finite temperature there are two scales, namely the natural nonperturbative length scale $1 /\left(\alpha_{W} T\right)$ and the (lattice) regulator or cutoff scale. If one assumes that the long wavelength phenomena are independent of the cutoff scale (which is still unclear) then dimensional analysis indicates the rate of Chern Simons number diffusion behaves parametrically as

$$
\Gamma \equiv \lim _{t \rightarrow \infty} \frac{\left\langle\left(N_{C S}(t)-N_{C S}(0)\right)^{2}\right\rangle}{V t}=\kappa\left(\alpha_{W} T\right)^{4}
$$

with $\kappa$ an unknown constant of order unity.

There are no reliable analytical estimates of $\kappa$, and at this time it appears that the only hopeful way of determining $\kappa$ is by numerical, lattice study of the classical theory, which should be a suitable analog theory in the infrared [6, 7, 8, 9, 10, 11, 12.

There has already been substantial work on finding $\kappa$ numerically [7, 13, 9, 14, 15, 10, 11]. All this work has been based on a lattice implementation of $N_{C S}$ as an integral over a local 
operator, usually

$$
2 \pi^{2} \frac{d N_{C S}}{d t}=\sum_{\text {sites } x} \sum_{\text {directions } i}\left[\left(\frac{E_{i}^{a}(x)+E_{i}^{a}(x-\hat{i})}{2}\right) \times \frac{1}{4} \sum_{\square_{j k}}\left(\frac{1}{2} \operatorname{Tr}-i \tau^{a} U_{\square_{j k}}\right)\right]
$$

(appropriate parallel transports implied) where the $E_{i}^{a}$ are the electric fields entering and leaving the point in the $i$ direction, and the latter sum is over the four plaquettes orthogonal to the $i$ direction and with a corner at the point $x$. That is, one takes a sum over lattice sites of $E \cdot B$, with $E$ and $B$ the average over neighboring space-time and space-space plaquettes of the Lie algebra content of the plaquette. This definition is chosen to reproduce $\left(g^{2} / 32 \pi^{2}\right) \int d^{4} x \operatorname{Tr} \tilde{F}^{\mu \nu} F_{\mu \nu}$ for smooth, slowly varying gauge fields, which it does. However, as a definition of the time derivative of $N_{C S}$ it has several drawbacks. First, it is not a total time derivative [14]. Second, it possesses white noise which grows as $1 / a$ (with $a$ the lattice spacing) [9]. It also gives a UV dominated, 'fake' contribution to the inferred diffusion of $N_{C S}$, which is clearly seen in the broken phase [11, 16, 17]. Finally, UV- dominated (tadpole) contributions from higher order terms in the expansion of $U$ may renormalize the inferred rate [10]. These drawbacks are serious, and until they are resolved we cannot have much confidence in the derived values of $\kappa$, particularly in the broken phase where the true rate will be smaller and any spurious UV generated diffusion could dominate.

We believe it is important to clear up these problems in order to get a valid determination of the efficiency of baryon number violation, which is simply related to $\kappa$ [18, 19]. In particular, it is not known whether $\kappa$ will depend on the physics of charge screening (hard thermal loops). The quantum theory has in addition to its natural nonperturbative length scale $l_{n} \propto 1 /\left(g^{2} T\right)$ a Debye screening length $l_{d} \propto 1 /(g T)$, and it has recently been argued that $\kappa$ should depend on the ratio of $l_{d}$ and $l_{n}$ as $\kappa \propto\left(l_{d} / l_{n}\right)^{2}$ [16, 20. Classical lattice simulations mimic hard thermal loop effects through interactions between the infrared modes and the short wavelength lattice modes [8], giving an effective screening length proportional to the square root of the lattice spacing. If hard thermal loops contribute as claimed, one should see $\kappa$ decrease linearly with lattice spacing. At present the numerical data argues against such a dependence [9, 14, but the evidence cannot be considered strong until the problems in the definition of $N_{C S}$ have been dealt with. Similarly, the problem of hard thermal loops in general [8] may be addressable by adding particle species which generate hard thermal loop effects to the classical simulations [21], but pursuing this project also demands a good definition of $N_{C S}$.

In this paper we present an alternative, topological definition of $N_{C S}$ on the lattice, related to that of Woit [22]. Its change as the system evolves smoothly from one configuration to another is strictly independent of small variations of the path between configurations, which prevents UV generated diffusion. It is also gauge invariant under small gauge transformations. The technique is to add a group-valued scalar field in the fundamental representation, which lives in the background of the gauge fields but does not influence their evolution. The role of this field is to track topology change in the gauge field, and $N_{C S}$ is to be associated with minus the winding number of the minimal energy configuration of this "slave" field. We outline the general idea in Section 2, and address the particulars of its lattice implementation in Section 3. In Section 1 we present numerical results for this technique in Yang-Mills and Yang-Mills Higgs theory. Section 5 concludes. 


\section{Slave field in the continuum}

Consider a continuum $\mathrm{SU}(2)$ spatial gauge field configuration $A_{i}^{a}(x)$. We want a surrogate for its Chern-Simons number $N_{C S}$ which will be easy to implement on the lattice. Our choice is to add a notional $\mathrm{SU}(2)$ valued scalar field $S(x)$. Under a gauge transformation $S(x)$ tranforms as $S(x) \rightarrow g(x) S(x)$. $S$ will be a "slave" field, meaning that it lives in the background of the gauge connection but it will not enter the dynamics of the gauge fields or any other physical fields in the problem. It may however be used to change the gauge in which we are working, in order to find the gauge in which $A_{i}^{a}(x)$ is smoothest.

Any $\mathrm{SU}(2)$ matrix may be expressed as $S_{11}=a, S_{12}=b, S_{22}=a^{*}, S_{21}=-b^{*}$, with $a=x^{0}+i x^{3}$ and $b=x^{2}+i x^{1}$, and $x^{i}$ a real four-component vector of unit length, $x^{i} x^{i}=1$. Thus the the slave field $S(x)$ provides a map from space to $\mathrm{SU}(2)=S^{3}$. The winding number of this map is

$$
N_{S}=\frac{1}{24 \pi^{2}} \int d^{3} x \epsilon^{i j k} \operatorname{Tr}\left(\partial_{i} S S^{\dagger} \partial_{j} S S^{\dagger} \partial_{k} S S^{\dagger}\right)
$$

which as we shall see may be very efficiently computed on the lattice. We shall give the slave field the Hamiltonian

$$
H(S)=\int d^{3} x \operatorname{Tr}\left(D_{i} S\right)^{\dagger}\left(D_{i} S\right)
$$

and note the following property: if the gauge connection is a vacuum (pure gauge) connection then the minimum energy configuration for the $S$ field has a winding number $N_{S}$ equal to minus the Chern-Simons number of the gauge field configuration. To see this, note first that in the gauge where $A_{i}^{a}=0$ everywhere, the minimum energy configuration for $S(x)$ is $S(x)=I$ everywhere (or any fixed element of $\mathrm{SU}(2)$ ). This configuration has zero winding number and zero energy. By gauge invariance, the minimum energy configuration for $S$ in any other vacuum configuration $A_{i}^{a}(x)$ must also have zero energy. If we gauge transform by $g(x)=S^{\dagger}(x)$, which carries $S(x)$ to $I$, the Hamiltonian then measures $\int A^{2}$, so the gauge field in this gauge must be $A_{i}^{a}(x)=0$ everywhere. Thus $S^{\dagger}(x)$ is the gauge transformation which carries $A_{i}(x)$ into the trivial vacuum. The Chern-Simons number of a vacuum gauge field is just the winding number of the gauge change which carries it to $A_{i}=0$, which in this case is the winding number of $S^{\dagger}(x)$; and $S(x)$ has minus this winding number, since $N_{S}$ is reversed by complex conjugation. Thus the winding number of the minimal energy configuration for $S(x)$ is $-N_{C S}(A)$.

On any compact space with smooth connection $A_{i}^{a}(x)$, it should always be possible to find a minimal energy slave field configuration (modulo a global SU(2) rotation), and we can take minus its winding number, $-N_{S}$, as our surrogate for Chern-Simons number?. By no means does $-N_{S}$ always equal $N_{C S}(A)$; in particular $N_{S}$ is always an integer, unlike $N_{C S}$; but it shares these important properties with $N_{C S}$ :

- it is a function only of the configuration and not of the path to the configuration,

- it is invariant under small gauge changes and changes by an integer, equal to the winding number of the gauge group element $g(x)$, under large ones,

\footnotetext{
${ }^{3}$ There could be multiple absolute minima but the definition would only be ambiguous in the unlikely event that two had different winding number.
} 
- it coincides with $N_{C S}$ for vacuum configurations.

Since we are only interested in the long time diffusion of $N_{C S}$ across the set of gauge equivalent configurations, we only care about the topological information specified by $N_{C S}$. And for this, $-N_{S}$ carries all of the appropriate information. One can make this argument rigorous by considering the case where one begins in vacuum, heats up the gauge fields and evolves them for a very long time before cooling down to vacuum again. In this situation the change in $-N_{S}$ would be precisely equal to the change in $N_{C S}$, and clearly in the long time limit the inferred diffusion rate would be independent of the initial heating and cooling times.

In practice the gauge fields $A_{i}(x)$ will evolve, and it is too much work to continually find the minimum energy solution for $S(x)$. It is more practical to give $S$ very efficient dissipative dynamics so that it will always remain close to an energy minimum. How will these dissipative dynamics behave when the gauge field configuration changes from one winding number vacuum to another? To get a general idea it is useful to consider the dissipative dynamics of $S(x)$ when it is in the wrong topological sector for the underlying gauge fields. For instance, suppose that $S(x)=I$ everywhere but the gauge field is in a winding 1 vacuum configuration. To see how $S(x)$ evolves, it is convenient to change gauge so that $A_{i}^{a}(x)=0$ but $S(x)$ is in a winding -1 configuration. This is just a standard nonlinear sigma model on $S^{3}$ in 3 dimensions, initially in a winding -1 configuration, and its evolution is is well known [23]. The winding configuration (or texture) is unstable to collapse; its potential energy decreases linearly with its size. So the winding in the slave field shrinks and concentrates until a singularity (a texture unwinding event) occurs. The winding then jumps discontinuously to the correct value, allowing the system to settle down to the correct minimum energy configuration. The important point to make is that there is no energy gap between topological sectors for the slave field; by making a nontrivial winding of arbitrarily small spatial extent one can get nonzero winding at arbitrarily little energy cost. Hence topology does not present an obstruction to the dissipative evolution finding the appropriate winding number, minimal energy configuration. Secondly, the winding number change occurs by the concentration of winding in a very small region and the development of a singularity.

What the slave field technique does is find the gauge transformation $S^{\dagger}(x)$ which minimizes $\int \vec{A}_{S}^{2}$ with respect to $S(x)$, where $\vec{A}_{S}$ is the gauge transform of $\vec{A}$ by the gauge group element $S^{\dagger}(x)$ (S(x) the slave field). We then take $N_{C S}$ to be the winding number of that gauge transformation. In doing so, we make the approximation that the Chern-Simons number of the configuration with minimal $\int \vec{A}^{2}$ is zero. Obviously this is not correct, but the value of $N_{C S}$ for this configuration should be modest and in particular will not grow without limit over the course of a Hamiltonian evolution of the gauge fields. Therefore, while the slave field definition will have some noise about the correct value of $N_{C S}$, due to the approximation just mentioned, the noise will have no diffusive power so the diffusion constant of $N_{C S}$ will be reproduced correctly by this procedure.

Another concern is that, by finding the slave field configuration through dissipative dynamics, we are not guaranteed to find the global minimum of $\int \vec{A}^{2}$ in the space of gauges, but only a local minimum. For nontrivial gauge fields there may be a Gribov ambiguity and multiple local extrema [24]. In our case this is only a problem if at least one of the extra extrema is a minimum (within the Gribov horizon) with a different winding number than 
the absolute minimum. This is possible. However, we do not expect that, in finite volume and with gauge fields which are smooth at short distances, such minima should exist with a large winding number difference from the global minimum. In this case the possibility of finding such a false minimum will increase the "noise" in this measurement of $N_{C S}$ (the typical difference between the measured winding number and the true Chern-Simons number now being the Chern-Simons number of the typical Gribov copy), but it will not lead to excess diffusive power during a long evolution of the gauge fields (since the size of the error will be bounded and will not grow with time). We will see this explicitly in the next section, when we compare this winding number measurement technique with an alternate "improved" technique due to Ambjorn and Krasnitz [17.

Finally, we mention another interesting consequence of the slave field transformation, which is that when the minimum energy configuration is found, $S(x)$ obeys $D_{i} D_{i} S=0$. Upon gauge transforming to $S(x)=I$, this becomes the Coulomb gauge condition $\partial_{i} A_{i}=0$. So the slave field technique is in fact just a way of determining the gauge change to Coulomb gauge.

\section{Implementing the slave field on the lattice}

We now turn to the question of implementing this idea on the lattice.

\section{1 defining the slave field and winding number}

It is clear how to define the slave field $S$ on the lattice. It will take on a value $S(x) \in \mathrm{SU}(2)$ at each lattice point which will transform under a gauge change as $S(x) \rightarrow g(x) S(x)$, and the parallel transport of $S(x)$ to the point $x-i$ will be $U_{i}(x-i) S(x)$. The simplest lattice Hamiltonian which gives the right continuum limit is

$$
H=\sum_{x, i}\left(1-\frac{1}{2} \operatorname{Tr} S^{\dagger}(x) U_{i}(x) S(x+i)\right)=\sum_{x, i}\left(1-\frac{1}{2} \operatorname{Tr} S^{\dagger}(x+i) U_{i}^{\dagger}(x) S(x)\right),
$$

and the winding number can be defined by a construction similar to that of Woit [22], as follows.

First we partition the lattice into unit cubes with lattice points as vertices. Each cube has a basepoint, and extends one unit in the $+x,+y$, and $+z$ directions from the basepoint; so the vertices of the cube are $(i, j, k),(i+1, j, k), \ldots(i+1, j+1, k+1)$ with $(i, j, k)$ the basepoint. A cube is called even if the sum of the coordinates of the basepoint is even and odd otherwise. Next we partition each cube into 5 tetrahedra, as shown in Figure 1. The triangulation is such that the faces of the tetrahedra match, i.e. we have represented three dimensional space as a simplicial complex. Consider the slave field $S(x)$ to take its values in the 3-sphere, using the identification of the last section. In each tetrahedron, we will interpolate the function $S(x)$ between its values on the vertices by a geodesic rule. For each pair of vertices on a tetrahedron we draw on the 3-sphere a great circle connecting the image points, and take $S(x)$ on the line connecting the two vertices to take on the values along the smaller arc of this great circle. Each face of a tetrahedron now has its edges map into a geodesic triangle on the 3-sphere; take the face of the tetrahedron to fill in the inside of the 
triangle (the geodesic triangle breaks the equatorial sphere defined by the 3 points into two regions, and we fill in the region of smaller area). Finally, the surface of each tetrahedron now encloses a geodesic tetrahedron on the 3-sphere; we take the interior of the tetrahedron to fill in the inside of this tetrahedron on the 3-sphere (the surface of the tetrahedron maps into a surface which breaks the 3-sphere into two regions, and we take the interior of the tetrahedron to fill in the smaller volume region). This construction is unique except for a set of measure zero (when the convex hull of the verticies of some tetrahedron contains the origin) and it is easy to determine the winding number of this interpolated $S(x)$.

The winding number of a map from some space (in this case the 3-torus on which the lattice lives) into the 3-sphere can be evaluated (for piecewise smooth maps which have nonzero Jacobian off a set of measure zero) by the following simple algorithm [22]; choose a point $q$ on the 3 -sphere, and find its inverse images. The winding number is the sum over inverse images of $q$ of the sign of the Jacobian at that point, i.e. the number of times $q$ is covered with positive orientation minus the number of times it is covered with negative orientation. In our case, this means that we must choose a point $q$ on the 3sphere, and for each tetrahedron we must determine whether the tetrahedron covers that point on the 3 -sphere, and if so with what orientation. If the four vertices $v_{1}, v_{2}, v_{3}, v_{4}$ of the tetrahedron map into the points $p_{1}, p_{2}, p_{3}, p_{4} \in S^{3}$ (note that $v_{i}$ must be positively oriented, i.e. $\left.\left(v_{4}-v_{1}\right) \cdot\left(\left(v_{1}-v_{2}\right) \times\left(v_{2}-v_{3}\right)\right)>0\right)$, then the orientation of the image of the tetrahedron is

$$
\operatorname{sign}\left(\epsilon_{i j k l} p_{1}^{i} p_{2}^{j} p_{3}^{k} p_{4}^{l}\right)=\text { orientation }
$$

and the point $q \in S^{3}$ lies inside the image of the tetrahedron if and only if

$$
\begin{aligned}
\operatorname{sign}\left(\epsilon_{i j k l} q^{i} p_{2}^{j} p_{3}^{k} p_{4}^{l}\right) & = \\
\operatorname{sign}\left(\epsilon_{i j k l} p_{1}^{i} q^{j} p_{3}^{k} p_{4}^{l}\right) & = \\
\operatorname{sign}\left(\epsilon_{i j k l} p_{1}^{i} p_{2}^{j} q^{k} p_{4}^{l}\right) & = \\
\operatorname{sign}\left(\epsilon_{i j k l} p_{1}^{i} p_{2}^{j} p_{3}^{k} q^{l}\right) & =\text { orientation }
\end{aligned}
$$

In practice, at single precision there is a slight risk that $q$ will lie on the boundary of one of the tetrahedra, and one of the above quantities will be zero. To cover this possibility we measure the winding number using more than one $q$ and check that they agree. In practice errors are very rare, but we retain the checking feature as a precaution.

\section{2 gauge dependence and gauge choice}

Next let us consider the behavior of this winding number. Suppose that the slave field is slowly varying, ie its value at any pair of vertices on a tetrahedron are separated by, say, less than $\pi / 2$ radians. Then moving the value of $S$ at a single point by some small amount (say, less than $\pi / 2$ radians) will cause the images of some tetrahedra to grow, at the expense of others; but the winding number will not change. To change winding number we must have one of the tetrahedra expand until it covers half the 3-sphere, at which point the geodesic rule switches which geodesic tetrahedron on the 3 -sphere it covers, and the winding number changes by \pm 1 . This will only occur if the values of the slave field at two neighboring points differ by a large amount. Hence we find that, if the initial slave field configuration is slowly 
varying, then the winding number will be invariant to small gauge transformations, meaning ones where the gauge element selected at each point is in the vicinity of the origin. This is the lattice analog of the invariance of the continuum winding number to small gauge transformations. Similarly, if the slave field is slowly varying, and we apply a large but slowly varying gauge transformation (ie $g$ need not be in a close neighborhood of the origin, but the angle between its values at neighboring points is always small), the winding number will change by the winding number of the gauge transformation. Note that both small gauge transformations and large but smooth gauge transformations may reduce the smoothness of the slave field, so a series of small gauge transformations can eventually change the winding number. Hence, the slave field winding number has properties much like what we need, but only when the slave field is kept slowly varying.

In general there will be two situations in which the slave field will not be slowly varying. One is avoidable, and the other is not. The first occurs if there are link matrices which are very far from the identity, in which case the slave fields on the two ends of the link will want to differ from each other by an angle equal to the angle by which the link differs from the identity. Since we are in three space dimensions, the thermodynamics of classical YangMills theory is super-renormalizable, and if our lattice spacing is small then the elementary plaquettes should all be close to the identity; so there should be some gauge which can prevent this from happening. We will discuss finding and staying in such a gauge below. The other circumstance in which the slave field varies rapidly in space is when the links are all close to the identity but there is a texture unwinding event in its final stages, i.e. a topological winding in the slave field has been concentrated into a very small volume. In the continuum such a winding is removed when the slave field develops a singularity; on the lattice what happens is that the winding reaches the lattice scale and then 'slips through the lattice', leading to a winding number change. This is our signal that the gauge field configuration has changed from being closer to one winding number vacuum to being closer to another; in this case we must allow the slave field to change winding number, and settle to the new $N_{C S}$ vacuum, before performing a large gauge transformation to make $S(x)$ uniform again.

From this discussion, we see that the slave field winding number will track $N_{C S}$ provided that we can find a gauge which is smooth (meaning all link matrices are rather close to the identity) at any moment and which does not change abruptly between timesteps (the equivalent of the requirement in the continuum case that the gauge is nonsingular). Temporal gauge (time links set to the identity) satisfies the latter requirement; the gauge field evolves smoothly in time because in this gauge

$$
U_{i}(x, t+\delta t)=\exp \left(i \delta t \tau \cdot E_{i}(x, t+\delta t / 2)\right) U_{i}(x, t)
$$

and $\delta t$ should be small (and so generally is $E$, which satisfies $E^{2} \sim 1 / \beta_{L}$ ). It is also possible to choose a gauge for the initial conditions in which the gauge field is smooth, by using the

\footnotetext{
${ }^{4}$ Note that this definition of smooth is very weak; in the continuum limit it does not require that the fields be differentiable or even continuous, but only that as the lattice spacing $a$ is made smaller, that $A$ grows slower than as $a^{-1}$. For classical fields, $A \sim a^{-1 / 2}$ at least in some gauge, and the connection becomes rapidly smoother, in the sense used here, as the lattice spacing is made smaller.

${ }^{5}$ Here and throughout our notation is the same as [9]. Our implementation of the equations of motion is that of [13], which differs at $O\left((\delta t)^{3}\right)$ from what is written here.
} 
freedom in temporal gauge to make a time independent gauge transformation. One can take for instance the lattice equivalent of Coulomb gauge [25], namely the gauge which minimizes

$$
\sum_{x, i}\left(1-\frac{1}{2} \operatorname{Tr} U_{i}(x)\right)
$$

This "lattice Coulomb" gauge should make the connection as smooth as possible. It is also the gauge in which the minimal energy slave field configuration is everywhere the identity. This gives a simple algorithm for finding this smoothest gauge; evolve the slave field dissipatively to an energy minimum, and then gauge transform by $g(x)=S^{\dagger}(x)$. Provided that the slave field was not in the middle of a texture collapse when we terminated the dissipative evolution, this should make the gauge connection smooth everywhere. And there are simple gauge invariant measurements which can tell if the slave field is close to a texture collapse event or some other phenomenon which will make the connections non-smooth in some small neighborhood. One can define a peak stress, for instance,

$$
\text { peak stress }=\sup _{x}\left[\sum_{i}\left(2-\frac{1}{2} \operatorname{Tr}\left(S^{\dagger}(x) U_{i}(x) S(x+i)\right)-\frac{1}{2} \operatorname{Tr}\left(S^{\dagger}(x) U_{i}^{\dagger}(x-i) S(x-i)\right)\right)\right] .
$$

If the peak stress exceeds some threshold then gauging to $S(x)=I$ will make the connection not smooth in some small neighborhood; otherwise the gauge $S(x)=I$ is smooth everywhere.

Evolving the system in temporal gauge indefinitely is insufficient, however, because the connections will gradually move away from being smooth. The condition $\vec{\nabla} \cdot \vec{A}=0$ is preserved by the evolution in temporal gauge only if $\vec{\nabla} \cdot \vec{E}=0$; but Gauss's law is $\vec{D} \cdot \vec{E}=0$, which only agrees at leading order. Eventually the connections will become far from smooth and the good behavior of the slave field winding number will break down. This is illustrated in Figure 2, which shows the evolution of the slave field winding number in the broken phase of Yang-Mills Higgs theory, on a $24^{3}$ lattice at $\beta_{L}=8$, starting in the gauge $S(x)=I$ and evolving the slave field with the efficient dissipative dynamics discussed below. The slave field winding number remains zero, as it should, for some time, but the connections and the minimum energy slave field configuration become ever less smooth, until the slave field winding number begins to oscillate wildly.

Instead it is necessary to make occasional gauge changes to restore the smoothness of the connection. As discussed earlier, to preserve the correct behavior of the slave field winding number these transforms must be either small or large but smooth, both in the sense defined earlier; and if the gauge transform is large, i.e. it changes the slave field winding number, then we must know the winding number before and after the gauge transformation, and add the change to a total count of windings removed by gauge changes. Our surrogate for $-N_{C S}$ is the current winding number plus the count of windings removed by past gauge changes.

If the (temporal gauge) evolution since the last gauge change has been relatively short and the slave field's peak stress was not large at the beginning of, during, or at the end of the evolution, then the gauge change to the gauge $S(x)=I$ will be small. If the slave field's peak stress was small at the beginning and end of the evolution but not in between, then the gauge change may be large, but it should be smooth (since the connection is smooth before and after). If on the other hand the slave field's peak stress is large at the end of the brief period of evolution, then the gauge change would probably not be smooth, and it will make 
the connections unsmooth in some small neighborhood; it is possible that making such gauge changes will cause us to lose information about winding number change. (For instance, if we applied a gauge change to $S(x)=I$ at every timestep, then unless the dissipative dynamics could fully collapse a texture event in a single timestep, we would never observe any change in its winding number; this is an example of a series of small gauge changes which change the winding number, because they bring the connections through non-smooth configurations in the process.) So our algorithm is to measure the slave field winding number every few (say 5) timesteps (remember that a time step is $\delta t$ times a lattice unit of time evolution; in all work in this paper $\delta t=0.05$ ), and provided that its peak stress falls below a threshold, we should apply a gauge transformation to the gauge $S(x)=I$ everywhere; but if the peak stress exceeds the threshold we should not change gauge, but should check again next time.

We have computed that, for our definition of peak stress, Eq. (11), if the plaquettes are all close to the identity, then the peak stress during a winding number changing evolution must exceed 2.0 at some time, and if we gauge change just before it first reaches 1.0 and again after it falls back to 1.0 we will always correctly identify the winding number change. These estimates are very conservative, and in practice, with smooth connections, the stress always peaks at at least 2.4 during texture collapse, and generally much higher (depending on how close the core of the texture is to a lattice site); and for the real case the ultraviolet fluctuations in the magnetic field also contribute to the peak stress, and the peak value is still higher. We have used a threshold of 1.2 in our work. We have verified for long evolutions that changing the threshold to 1.1 or 1.3 does not change the determined winding number, although it is also clear that a sufficiently high or low threshold would either allow true winding number changes to be gauged away, or prevent smoothing gauge changes for long enough that the configuration becomes non-smooth, in which case the winding number is sensitive to small changes in the configuration and loses its topological interpretation.

\section{3 dissipative evolution algorithm}

All that remains is to specify an efficient dissipative algorithm for keeping the slave field near the minimal energy configuration while the gauge fields evolve. An element of a simple quench algorithm developed by Mandula and Ogilvie [25] is to minimize the Hamiltonian with respect to variations in the slave field at one point, $S(x)$. That is, we replace $S(x)$ with the value which minimizes

$$
\sum_{i}\left(2-\frac{1}{2} \operatorname{Tr}\left(S^{\dagger}(x) U_{i}^{\dagger}(x-i) S(x-i)\right)-\frac{1}{2} \operatorname{Tr}\left(S^{\dagger}(x) U_{i}(x) S(x+i)\right)\right),
$$

which is minimized by choosing $S(x)$ to be the sum of the parallel transports of the nearest neighbors, treated as points in $\Re^{4}$, projected to unit modulus. (Note that the length of the vector in $\Re^{4}$ before the projection is 6 minus the "stress" at that point, which is then a biproduct of the calculation.) A quench of the slave field consists of sweeping over lattice sites in some order, applying this algorithm at each. The updates of even and odd sites are independent, so it is easiest to alternately update all even and odd sites. A weak sinusoidal perturbation to $S(x)$, with wave number $k$ and lattice dispersion measure $\omega^{2}=\sum_{i} 4 \sin ^{2}\left(k_{i} / 2\right)$, will be suppressed by a factor of $\left(1-\omega^{2} / 12\right)^{2}$ for each update of all sites, so the quench is very efficient at removing high $k$ excitations but less effective in the infrared. 
The simplest quench algorithm is to first take $S(x, t+\delta t)=S(x, t)$ and then apply the above quench once (or several times) each timestep. This can be significantly improved by using "memory", or giving the system damped inertial dynamics. Since the timestep is much shorter than any inverse frequency in the system's dynamics, the update of $S$ at one timestep will be almost the same as for the previous one; so we apply $m<1$ times the last time's update pre-emptively before quenching. In other words, rather than taking $S(x, t+\delta t)=S(x, t)$ and then quenching, we take $S(x, t+\delta t)=\left(S(x, t) S^{\dagger}(x, t-\delta t)\right)^{m} S(x, t)$ as our initial guess and then quench. Here $S$ at previous timesteps is always the value after the quench was applied at that timestep. The quantity $S(x, t) S^{\dagger}(x, t-\delta t)$ can be viewed as the slave field's momentum and is stored as a Lie algebra element (so taking the $m$ power becomes multiplication by $m)$. $(1-m)$ acts as a damping coeffient and should be positive for stability; we find $1-m=\delta t$ proves quite efficient, increasing the performance of the algorithm, in the absence of a collapse event in its final stages, by on order $1 / \delta t$.

Two further modifications are wise, because of the "texture collapse" events. In such an event the slave field changes very rapidly as it removes the unwanted winding, after which the direction it needs to evolve abruptly changes. We prevent rebounding after the collapse event by turning down or off the memory effect at sites where $S(x, t) S^{\dagger}(x, t-\delta t)$ is too far from the identity, say $1-(1 / 2) \operatorname{Tr} S(x, t) S^{\dagger}(x, t-\delta t)>(1-m)^{2}$. The slave field then has fast, damped inertial dynamics with nonlinear damping coefficient. Also, to make the final collapse of the textures more efficient (and to minimize the time spent with the peak stress above the threshold), whenever the peak stress goes above its threshold we triple the number of quenches used, and substitute $m^{3}$ for $m$, in a neighborhood of the most stressed point.

We have run several tests of this quench algorithm. We have evolved two slave fields simultaneously on the same gauge field background, taken from a real simulation, single quenching one and double quenching the other; we find almost no difference between how well they minimize the slave field energy, and the double quenched slave field typically discovers winding number changes only slightly sooner (at most 1 lattice length of time, or 20 updates) than the single quenched slave field. We also evolved two slave fields simultaneously, each single quenched, but beginning one of them from the minimal energy configuration and the other from a completely random initial configuration. Within one lattice unit of time their energies were comparable and their winding numbers differed by at most 2 , and after 5 lattice units of time their winding numbers ceased ever to differ and they became essentially identical.

\section{Numerical results}

Here we present results of numerical investigations of the motion of $N_{C S}$ using the slave field technique.

\section{1 tests}

First we tested the slave field technique on "mocked up" backgrounds to see that it behaves as expected. In the naive vacuum, starting the slave field in a winding 1 configuration and applying the dissipative algorithm, we observe the slave field energy to drop while the peak 
stress increases, peaking at a value always above 2.4, at which point the winding number changes to zero. The energy and peak stress then fall off. This is the behavior expected for a "texture collapse" event. We have also "mocked up" a Sphaleron transition; we find an $N_{C S}=1$ vacuum gauge field configuration by gauging away a winding one slave field configuration with initially flat connection; we then take the $n$ 'th root of each gauge link and "evolve to" the winding 1 gauge field configuration by starting with flat connections and repeatedly multiplying each link by the appropriate $n$ 'th root. The slave field evolves dissipatively in this background. It changes winding number slightly more than halfway through the evolution, shortly after the underlying configuration switches from being nearer one vacuum configuration to being nearer the other. As we conduct the evolution in more steps, the slave field changes winding number more steps, but a smaller fraction of the length of the evolution, after the midpoint of the evolution. The response of the slave field to a change in the winding number of the underlying configuration is delayed, but not very much; the sluggishness to respond to winding number change will translate in a real simulation with a timestep $\delta t=0.05$ to a delay of about one lattice unit of time, which is small. We also observe that the "old" definition of Chern-Simons number systematically underestimates the actual $N_{C S}$ change through this evolution, by an amount which grows worse as the initial winding becomes less spread out. This is the result of nonrenormalizable operator corrections and we will discuss it again below.

\section{2 comparison with the cooled field technique}

Next, we want to see if the slave field method will be reliable for tracking $N_{C S}$ in real simulations of Yang-Mills or Yang-Mills Higgs theory. It would be illuminating to compare it against the "old" definition, Eq. (3), except that we strongly believe that this definition is contaminated by lattice artifact diffusion. However, Ambjørn and Krasnitz have recently proposed a patch for this definition which should eliminate almost all of its white noise and lattice artifact diffusion [17]. The idea is to copy the connections at each timestep and quench or "cool" them, and then to track $N_{C S}$ for the evolution of these cooled fields. The infrared fields, which are responsible for the diffusion of $N_{C S}$, will be unaffected, but the ultraviolet excitations on top of them will be removed, and will no longer contribute white noise, lattice artifact diffusion, or screening of the field operators in the old definition of $N_{C S}$, Eq. (3). The cooling is just an evolution of fixed length under straight dissipative dynamics, $\dot{U}=-\partial H / \partial U$ (correctly interpreted for group elements).

We implemented this algorithm with a cooling of length of 1.25 lattice units (which should be enough to remove the worst behaved frequencies and greatly ameliorate the problems with the old definition, although a longer cooling might be preferable). We then evolved Yang-Mills Higgs theory in the symmetric phase, just at the phase transition temperature, recording $N_{C S}$ using all three definitions; the results are presented in Figure 3. These data were taken on a $24^{3}$ grid at $\beta_{L}=8.07, \lambda_{L}=0.2$, and $m_{H L}^{2}=-0.3223$ (bare), the same values generally used in [11]; we also use the thermalization and evolution algorithms and the definition of temperature used there. The total length of the evolution was 10500 lattice units. The two new definitions of $N_{C S}$, the slave field definition and the cooled field definition, agree very closely, though there is considerable high frequency noise. Some of this noise might arise because the cooling step in the cooled field approach changes $N_{C S}$, 
but probably most of it is due to the problems with the slave field definition which were discussed at the end of Section 2.

To study how well these methods agree and what diffusion constant they imply, we apply a cosine transform and study their frequency spectra ${ }^{\circ}$. Writing $N_{C S}$ as a function of time as $z(t)$, we define

$$
\tilde{z}(m)=\int_{0}^{t_{f}} \frac{d t}{t_{f}} z(t) \cos \left(\frac{m \pi t}{t_{f}}\right)
$$

and find that, if $z(t)$ evolves diffusively with diffusion constant $\left\langle\left(z(t)-z\left(t^{\prime}\right)\right)^{2}\right\rangle=\Gamma\left|t-t^{\prime}\right|$, then $\tilde{z}(m)$ will be Gaussian distributed and independent, with variance

$$
\langle\tilde{z}(m) \tilde{z}(n)\rangle=\frac{\Gamma t_{f}}{2 \pi^{2} m^{2}} \delta_{m n}
$$

When the sampling is discrete with $N$ intervals between measurements, then the integral should be replaced by a sum, with $z$ at each endpoint only sampled with half weight; in this case $m$ on the rhs. of Eq. (14) should be replaced with $(2 N / \pi) \sin (m \pi / 2 N)$. The addition of white noise will add an $m$ independent term, but the transform coefficients remain independent and Gaussian, which makes this decomposition particularly convenient for analysis. Other sorts of noise could make the Fourier components nonGaussian or correlated, but this will not be a problem if we only use the most infrared Fourier modes in the analysis, since on long time scales the evolution should be almost purely diffusive.

We use this technique to compare the slave field, cooled field, and "old" definitions of $N_{C S}$. Denote their cosine transform spectra as $\tilde{z}_{s}, \tilde{z}_{c}$, and $\tilde{z}_{o}$ respectively. We will be concerned only with the infrared, since we want to know how they track $N_{C S}$ over long times and if there are noise contributions to its diffusion constant, so we will consider sums over the first several terms, reweighted by multiplying each $z$ by $m$ so as not to overemphasize the first few coefficients.

First, let us assume that the cooled field reports only $N_{C S}$ but that it has a multiplicative renormalization, because of nonrenormalizable operators and screening from the modes which were not completely quenched. The slave field method, being topological, should track $N_{C S}$ unrenormalized, but perhaps with some extra diffusive signal if there are occasional erroneous identifications of winding number change. We can find the multiplicative renormalization of the cooled field technique by measuring $\tilde{z}_{c} \cdot \tilde{z}_{s} / \tilde{z}_{c}^{2}$, where

$$
\tilde{z}_{1} \cdot \tilde{z}_{2} \equiv \sum_{n=1}^{N} m^{2} \tilde{z}_{1}(m) \tilde{z}_{2}(m) \text {. }
$$

The noise part of $\tilde{z}_{s}$ is uncorrelated and will not contribute (on average), and we will just find the inverse of the renormalization of the cooled field definition. The result, using the first 25 to 50 transform coefficients, is $\tilde{z}_{c} \cdot \tilde{z}_{s} / \tilde{z}_{c}^{2}=1.05 \pm 0.01$ (the error bar reflecting dependence on the number of points used). Hence, the cooled field technique underreports winding number

\footnotetext{
${ }^{6}$ In [1] we advocated using a sine transform. That approach is not time symmetric, and when white noise is present, the noise in the first datapoint is propagated into all the data. The cosine transform used here gives correct performance in the presence of white noise.
} 
change by a factor of 0.95 , for this level of cooling and this lattice spacing].

We can also see how large the noise in the slave field definition is from $1-\left(\tilde{z}_{c} \cdot \tilde{z}_{s}\right)^{2} /\left(\tilde{z}_{c}^{2} \tilde{z}_{s}^{2}\right)$, which measures the diffusive power in the slave field definition which is not correlated with the cooled field definition (and must be noise, in one definition or the other). The value, using $N=25$, was 0.002 ; except for the multiplicative renormalization of the cooled field technique, the two methods agree in the infrared almost exactly. In fact, the difference is too small to be explained even by one accidental miscounting of a winding number change by the slave field technique, as can be seen in Figure 4 . The difference between the definitions is larger at higher frequencies; if we had used a much larger value of $N$ above we would have found poorer agreement. This represents non-diffusive noise in the slave field definition, presumably due to the algorithm picking Gribov copies with different winding number from the one which absolutely minimizes the slave field energy. We can see from Figure th that the slave field winding number is often off by \pm 2 from the cooled field value, but never for long, and the moving averages are in excellent agreement. Also note that it is possible that the cooled field technique has some residual spurious diffusion, because the ultraviolet modes are not completely cooled; but the quality of the agreement between the slave and cooled field methods in the infrared indicates that even with a relatively modest cooling depth of 1.25 lattice units, spurious UV contributions to the diffusion constant for the smoothed field definition are very small.

It is also instructive to apply these analyses to see how accurate the "old" definition of $N_{C S}$ is, taking the slave field definition as a benchmark. for this run, using the first 40 transform coefficients, we find $\tilde{z}_{o} \cdot \tilde{z}_{s} / \tilde{z}_{s}^{2}=0.67 \pm .06$ and $1-\left(\tilde{z}_{o} \cdot \tilde{z}_{s}\right)^{2} /\left(\tilde{z}_{o}^{2} \tilde{z}_{s}^{2}\right)=0.28 \pm .06$. The renormalization of the old definition is startlingly large, and there is considerable diffusive power uncorrelated with true topology change. (The error bars are estimates based on the expected RMS "accidental" projection of the noise part of the old definition of $N_{C S}$ along the slave field definition, and the expected statistical fluctuations in the size of the noise part, $1 / \sqrt{N}$ of its amplitude.)

Another problem we can address with the slave field technique is the diffusion rate of $N_{C S}$ in the broken electroweak phase. To do this we cooled and then heated the starting configuration for the symmetric phase run discussed above, to bring it into the broken electroweak phase at exactly the same temperature. We then made another Hamiltonian evolution of 11000 lattice units length. The slave field winding number and the old definition of $N_{C S}$ for this run are presented in Figure 5; while the old definition of $N_{C S}$ indicates considerable diffusion, the slave field technique shows that not a single winding number change occurred. This confirms the conjecture [1] that the diffusion observed in the broken electroweak phase using the old definition of $N_{C S}$ is a lattice artifact, and it also agrees with the expectations from the semiclassical "Sphaleron" calculation that the rate should be exponentially small [5]. In particular this run bounds the diffusion constant for these values of parameters ( the broken phase here has $\phi=1.4 g T$ ) to be $\kappa<0.002$, though the error bar here is not Gaussian.

\footnotetext{
${ }^{7}$ This under-reporting could be ameliorated by cooling more heavily and using an improved local operator free of $O\left(a^{2}\right)$ errors for $E \cdot B$, and by going to a finer lattice. We do not view it as a fundamental defect of the cooled field method.
} 


\section{3 lattice spacing dependence}

Finally we will investigate how the diffusion constant for $N_{C S}$ scales with lattice spacing. In Yang-Mills Higgs theory in the symmetric phase the rate will depend weakly on the distance from the phase transition temperature and on the scalar self-coupling. The physically interesting diffusion rate will be the rate in Yang-Mills Higgs theory in the symmetric phase with some supercooling, at a value of the scalar self-coupling which is not yet known. However, we are interested here only in the lattice spacing dependence of the rate and these other problems only complicate matters, so we analyze pure SU(2) Yang-Mills theory.

Before presenting the results we should point out that even if the rate of $N_{C S}$ diffusion is independent of the strength of charge screening, we should still expect a weak lattice spacing dependence in our results if we translate the diffusion rate per unit volume in lattice units into the diffusion rate per unit physical volume using the tree level relation $\beta_{L}=4 /\left(g^{2} a T\right)$. As discussed in [26], the wave function normalization on the lattice will receive $O\left(\beta_{L}^{-1}\right)$ corrections because the ultraviolet modes screen the infrared physics differently on the lattice than in the continuum; these corrections can be understood as a correction in the matching between the lattice length scale and the physical length scale. There is also a correction to the infrared thermodynamics from the $A_{0}$ fields which depends on the Debye mass, which varies with lattice spacing in $\mathrm{SU}(2)$ Yang-Mills theory with the Kogut-Susskind Hamiltonian used here as

$$
m_{D}^{2}=\frac{4 g^{2} \Sigma T}{4 \pi a}=\frac{\Sigma \beta_{L}}{4 \pi} g^{4} T^{2} .
$$

The former correction can be extracted from results in [26] by setting $\tan \Theta_{W}=0$, dropping all scalar contributions, and using the results in Appendix $\mathrm{C}$ of that paper to include the $A_{0}$ field; we find that the lattice volume should be converted into physical volume by using

$$
\beta_{L, \mathrm{imp}}=\beta_{L}-\left[\frac{70}{3} \frac{\xi}{4 \pi}+\frac{1}{3} \frac{\Sigma}{4 \pi}+\frac{1}{3}\right]=\beta_{L}-0.701 \quad(\xi=0.152859325)
$$

in place of $\beta_{L}$ in the formula for $a$. This gives the correct relation to a three dimensional continuum theory which, however, has a wrong (and lattice spacing dependent) Debye mass. To correct for the different Debye mass it is best to express the final answer in terms of $\bar{g}_{3}^{2}$, the natural inverse length scale of the 3 dimensional theory with the $A_{0}$ field integrated out. $\bar{g}_{3}^{2}$ sets the scale for nonperturbative infrared physics. It is related to $g^{2}$ by [27]

$$
\bar{g}_{3}^{2}=g^{2} T\left(1-\frac{g^{2} T}{24 \pi m_{D}}\right)
$$

This settles how to minimize errors in converting spatial lengths between the lattice and the continuum; but the corrections to the rescaling of the time direction need not be the same. This rescaling involves ultraviolet lattice artifacts in the real time evolution of the lattice system, which have not yet been studied in the literature. We will not solve this problem here, but we will make an educated guess, based on reasoning presented in

\footnotetext{
${ }^{8}$ Note that an early preprint version of 26 contained an algebraic error, producing a slightly different expression. Here we use the corrected version of that paper.
} 
Appendix A, that the $O(a)$ correction should be half as large as for a spatial direction. The final relation between $\Gamma$, the diffusion constant for $N_{C S}$ in lattice units, and $\bar{\kappa}$, the diffusion constant in terms of the characteristic length scale $1 / \bar{g}_{3}^{2}$ for nonperturbative physics, is then

$$
\left.\bar{\kappa} \equiv \Gamma \text { (phys. units) }\left(\frac{4 \pi}{\bar{g}_{3}^{2}}\right)^{4}=\Gamma \text { (lattice units }\right)\left(\pi \beta_{L}\right)^{4}\left(\frac{\beta_{L, \text { imp }}}{\beta_{L}}\right)^{3.5}\left(1-\frac{1}{6 \sqrt{4 \pi \Sigma \beta_{L}}}\right)^{-4} .
$$

We will present our results in terms of this $\bar{\kappa}$, and as $\kappa=\Gamma$ (lattice units) $\left(\pi \beta_{L}\right)^{4}$ (without thermodynamic corrections) for comparison with other literature.

We are interested here in finding the large volume limit of $\bar{\kappa}$ at several lattice spacings. As we have seen, the old definition of $N_{C S}$ is predominantly signal with a smaller, UV dominated noise component; so studies of the volume dependence of the diffusion constant, using the old definition, should reliably identify what a sufficient volume to achieve the large volume limit is. Ambjørn and Krasnitz used this definition and found that when the lattice exceeds $2 \beta_{L}$ points on a side, the rate has achieved a large volume limit [9]. We use a lattice $3 \beta_{L}$ on a side, which should be abundantly sufficient, and we use this same physical volume for each lattice spacing to prevent any systematic differences between lattice spacings due to residual lattice volume dependence. We have studied lattices with $\beta_{L}=6,8,10,12$, and 16 .

If $N_{C S}$ followed a perfect random walk then we could assume that the cosine transform coefficients $\tilde{z}(m)$ were Gaussian with variance $\left\langle\tilde{z}^{2}(m)\right\rangle=A / m^{2}$ and convert $A$ into the diffusion constant. But realistically the motion of $N_{C S}$ will only be approximately diffusive on long time scales, with corrections at finite $m$. If we had a lot of data then we could fit just the very low frequency coefficients to such a form, but to get good statistics it is necessary to make a fit of a larger number of coefficients with some assumption about the noise. At the same time we want to make sure that systematic errors in the fitting procedure are smaller than statistical errors. At finite $m$ we expect that $\tilde{z}(m)$ should become weakly nonGaussian, that there should be small cross-correlations between different $\tilde{z}(m)$, and that the variance $\left\langle\tilde{z}^{2}(m)\right\rangle$ should receive corrections from the $A / m^{2}$ form. If we only study the values of $\tilde{z}^{2}(m)$ then only the latter needs accounting for to find the diffusion coefficient. The first nontrivial correction which can occur at small $m$ is $\left\langle\tilde{z}^{2}(m)\right\rangle=A / m^{2}+B$. With this in mind we fit the first several cosine transform coefficients to $m^{2} \tilde{z}^{2}=A+B m^{2}$. We vary the number of coefficients used and find the point where the residuals first show a trend or the fit becomes poor, or the $B m^{2}$ term becomes comparable to the $A$ term for the largest $m$; we then fit using half this many coeffecients, to be sure that the fitting Ansatz is still applicable. We have found that both $A$ and its error are weakly dependent on where we place the cut, and we have also checked the technique with blind tests on artificially generated (diffusion plus noise) data. Note that the Ansatz we use for the behavior of $N_{C S}$ is that it is a Brownian signal plus noise which is white at least on long time scales. This is the same assumption made in the fitting procedure used by Ambjorn and Krasnitz [9, 17].

The results, presented in Table 1 and Figure 0 , distinctly show nonvanishing dependence on lattice spacing in the diffusion rate per unit physical 4-volume. However, the dependence is not as large as predicted by Arnold, Son, and Yaffe [16], who argue that the data should be proportional to $\beta_{L}^{-1}$ (proportional to $a$ ).

It is possible that the argument of Arnold, Son and Yaffe is correct in the strict $\alpha_{W} \rightarrow 0$ (for our purposes, $\beta_{L} \rightarrow \infty$ ) limit, but that the lattices investigated represent an intermediate 


\begin{tabular}{|c|c|c|c|c|c|}
\hline Phase & $\beta_{L}$ & lattice 4-volume & Def. of $N_{C S}$ & $\kappa$ & $\bar{\kappa}$ \\
\hline broken & 8.07 & $24^{3} \times 11000$ & old & $0.15 \pm 0.01$ & $0.11 \pm 0.01$ \\
\hline broken & 8.07 & $24^{3} \times 11000$ & slave & $0.000 \pm 0.003$ & $0.000 \pm 0.002$ \\
\hline symmetric & 8.07 & $24^{3} \times 10500$ & old & $0.93 \pm 0.05$ & $0.70 \pm 0.04$ \\
\hline symmetric & 8.07 & $24^{3} \times 10500$ & cooled & $1.41 \pm 0.13$ & $1.07 \pm 0.10$ \\
\hline symmetric & 8.07 & $24^{3} \times 10500$ & slave & $1.57 \pm 0.14$ & $1.19 \pm 0.11$ \\
\hline Yang-Mills & 6 & $16^{3} \times 32000$ & slave & $2.61 \pm 0.18$ & $1.77 \pm 0.12$ \\
\hline Yang-Mills & 8 & $24^{3} \times 33000$ & slave & $2.25 \pm 0.19$ & $1.70 \pm 0.14$ \\
\hline Yang-Mills & 10 & $30^{3} \times 50000$ & slave & $1.91 \pm 0.12$ & $1.53 \pm 0.10$ \\
\hline Yang-Mills & 12 & $36^{3} \times 60000$ & slave & $1.75 \pm 0.12$ & $1.46 \pm 0.10$ \\
\hline Yang-Mills & 16 & $48^{3} \times 75000$ & slave & $1.34 \pm 0.10$ & $1.18 \pm 0.09$ \\
\hline
\end{tabular}

Table 1: Results for $\kappa$ (naive lattice continuum relation) and $\bar{\kappa}$ (relation including thermodynamics corrections) in Yang-Mills theory at several lattice spacings, and for Yang-Mills Higgs theory in each phase and for three measurement techniques for $N_{C S}$.

value where their approximations do not fully apply. One might expect for instance that there are corrections which depend on the ratio of $l_{d}$, the Debye length, and $l_{n}$, some characteristic nonperturbative length scale, leading to order $\left(l_{d} / l_{n}\right)^{2} \propto \beta_{L}^{-1} \sim a$ corrections to a $\kappa \propto \beta_{L}^{-1}$ scaling law. To illustrate this, we plot $\beta_{L} \kappa$ against $\beta_{L}^{-1}$ (lattice spacing), in Figure 7 . The argument of Arnold, Son, and Yaffe is that such a plot should give a curve with a finite $y$ intercept; but there may be a nonzero slope in the approach to this point. For illustrative purposes we fit our data, both with an without thermodynamic corrections, to a straight line. The fit of the data before thermodynamic corrections has a smaller slope, but this does not mean that the thermodynamic corrections are wrong; there could be both a non-negligible $O(a)$ thermodynamic correction and an $O(a)$ correction to the Arnold Son Yaffe scaling law, which are of opposite sign. The thermodynamically corrected data do not exclude the Arnold Son Yaffe scaling law, but they require that there are quite large finite $\left(l_{d} / l_{n}\right)^{2}$ corrections. Note that $l_{d}$ of the lattice Yang-Mills system equals the value in the physical quantum system, $m_{D}^{2}=11 g^{2} T^{2} / 6$, at $\beta_{L} \simeq 17$, if one uses the physical value of $g^{2}$; so the strength of hard thermal loop effects in the finest lattices used are approaching the physically interesting values. The fit from Figure 7 suggests about a $20 \%$ finite $\left(l_{d} / l_{n}\right)^{2}$ correction from the $\alpha_{W}^{5}$ scaling rule at this value, but the difference between the lattice and continuum hard thermal loops means that we cannot directly use the lattice value of either this correction, or of the coefficient for the $\alpha_{W}^{5}$ scaling law, to give the continuum theory value. As for verifying whether the argument of Arnold, Son, and Yaffe is correct, our results appear consistent with their argument but demand substantial finite $\left(l_{d} / l_{n}\right)^{2}$ corrections.

It is difficult to view the data as consistent with a finite limit to the classical Sphaleron rate in the $a \rightarrow 0$ limit, ie with an $\alpha_{W}^{4}$ scaling rule, becase if the ultraviolet physics is unimportant to the infrared dynamics (the assumption which gives an $\alpha^{4}$ scaling rule) then it is hard to see why there should be very large $O(a)$ dynamical corrections, which Figure 6 clearly requires. 


\section{Conclusion}

We have demonstrated a topological solution to the problem of tracking Chern-Simons number in classical, real time lattice evolutions. Our technique suffers from fairly substantial noise, but this noise is white at least on long time scales and does not influence the extracted Chern-Simons number diffusion constant per unit physical 4-volume.

We have used our technique to study the diffusion constant for $N_{C S}$ in the broken electroweak phase and find that the rate is very substantially suppressed with respect to the symmetric phase rate; for the Higgs self-coupling we used, the rate at the equilibrium temperature is consistent with zero and is less than $\kappa=0.005$, in agreement with the expectations of the "Sphaleron approximation" estimate of the rate but in contradiction to previous results, which were contaminated by ultraviolet diffusion due to the non-topological definition of $N_{C S}$ used there [10]. We have also measured the diffusion constant in Yang-Mills theory at a range of lattice spacings, using an $O(a)$ improved match between the lattice spacing and the physical length scale, and we find a nonzero dependence on lattice spacing, which implies that the infrared dynamics relevant to $N_{C S}$ diffusion depends in an important way on the physics of hard thermal loops. This contradicts the results of [9, 14] because the definition of $N_{C S}$ used there is screened by ultraviolet modes, and undermeasures true topology change by a $\beta_{L}$ dependent amount. The strength of the $\beta_{L}$ dependence in our data is weaker than that predicted by Arnold, Son, and Yaffe [16, 20], but it is consistent with their proposal if there are substantial finite $\left(l_{d} / l_{n}\right)^{2}$ corrections.

We will end by mentioning some limitations of the slave field technique developed here, and we will comment on their possible resolution. We will also comment briefly on the classical lattice approach in general, in light of these results.

The most serious defect of the technique as presented here is that it breaks down as the lattice volume becomes too large. The reason is that we have advocated not applying a gauge change, anywhere, whenever the slave field is under too much stress at any one point. As the volume increases, it will become ever more likely that one such site is present somewhere in the volume; at sufficient volume there will generically be a texture collapse event in its final stages, somewhere in the volume, at any one time. This will prevent gauge changes from ever being made, or at least they will become very rare; but as we have discussed, the gauge will then drift away from being smooth and the winding number determination will become unstable. In a large volume it is necessary, then, to make gauge transformations which do not change the gauge very near places with high slave field stress, but gauge to slave field equals identity far away, and smoothly interpolate in between. We have not yet implemented this idea.

The other defect of the slave field technique as presented here is the approximation that the configuration in which the minimal energy slave field is everywhere the identity has $N_{C S}=0$. The white noise we discussed is essentially due to ignoring the $N_{C S}$ of this configuration. This defect could be greatly ameliorated by making a reasonably accurate estimate of $N_{C S}$ for this configuration, every time it is desired to write out the current value of $N_{C S}$. While this change would not alter the diffusion constant of $N_{C S}$ measured, it might improve the statistics of the determination, since shorter time scale diffusive motion could be seen over white noise and included in the statistics. Note that the technique for estimating $N_{C S}$ could be reasonably numerically expensive without affecting performance, since it need 
only be applied every time we wish to read out $N_{C S}$, not every time step; in particular it might be easier to find $N_{C S}$ of a cooled version of the configuration in question.

Finally, we should remark that since the classical, lattice rate of $N_{C S}$ diffusion is lattice spacing dependent, it is a difficult problem to relate the lattice results to the rate in the physical, quantum theory. The complications have been outlined recently in 28. Probably to find the rate applicable to the quantum theory, we will have to improve the lattice simulation in some way which properly reproduces the hard thermal loop effects, a possibility foreseen in [8]. For instance, one could add "particle" degrees of freedom, as suggested in [21]. Almost any practical, gauge invariant method will almost certainly need to be formulated in terms of lattice link variables, and in this case $N_{C S}$ can be tracked topologically using the slave field method presented here.

\section{Acknowledgements}

We wish to thank Peter Arnold, Chris Barnes, Martin Bucher, Alex Krasnitz, Anthony Phillips, and Michael Shaposhnikov for useful conversations or correspondence. This research was funded in part by a PPARC (UK) rolling grant, by a start-up grant provided to NT by Cambridge University, and by the support of HEFCE (UK) for the Cosmology Computing Center. GM was supported under NSF contract NSF - PHY96-00258. Research was conducted in cooperation with Silicon Graphics/Cray Research utilizing the Origin 2000 supercomputer.

\section{A Lattice and continuum time scales}

In this appendix we estimate (but do not compute) the $O(a)$ improved matching condition between the lattice and continuum time scales.

First observe that there is a simple argument which almost exactly explains the rescaling which was necessary for the spatial length scales. The most serious difference between the lattice and continuum implementations of the gauge theory is that the noncompact $A$ fields are replaced with compact link matrices $U$. The matrix $U$ is not $1+i \tau \cdot A$ but $1+i \tau \cdot A-A^{2} / 2-i \tau \cdot A A^{2} / 6+\ldots$, and these higher terms generate an infinite set of extra (lattice artifact) interaction terms. There are also derivative corrections to all interaction terms which only appear in the lattice theory. These properties of the lattice theory are actually inevitable in any implementation which is gauge invariant with a local Lagrangian. They lead to extra diagrams in the perturbation expansion of the lattice theory and substantial corrections in the values found for diagrams which do have continuum analogs, leading to substantial differences between the ultraviolet renormalizations of the two theories (lattice and continuum), which must be corrected for in the Lagrangian of the lattice theory. In the case of 3 dimensional gauge theory, these corrections vanish at least linearly in $a$; the $O(a)$ corrections arise at one loop in lattice perturbation theory and are computed in [26]. There is a very simple mean field theory argument, due to Lepage and Mackenzie [29], which allows a quick and startlingly accurate estimate of their size, namely the "tadpole improvement"

technique. The idea is to guess that the correction can be absorbed by rescaling each link matrix by a constant factor equal to the $-1 / 4$ root of $\langle 1 / 2 \operatorname{Tr} \square\rangle$, the average plaquette divided by its vacuum value. To see how well this approximation works, compare the $O(a)$ 


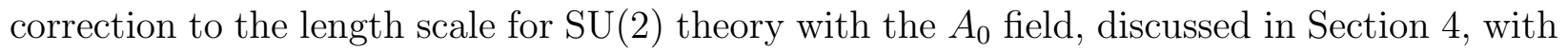
the tadpole estimate:

$$
\beta_{L, \text { imp }}=\beta_{L}-0.701, \quad \beta_{L, \text { imp }}(\text { tadpole estimate })=\beta_{L}-1.000 .
$$

Similarly, in the pure compact Abelian theory, the one loop correction (with or without the $A_{0}$ field, since it does not interact) is $\beta_{L \text {,imp }}=\beta_{L}-1 / 3$, which is exactly the tadpole prediction.

We will simply assume that the dominant contribution to the rescaling of the time scale also arises from tadpoles. Since tadpole contributions appear already in the abelian theory, we can look at this (much simpler) case to try to understand the rescaling of the time scale.

In the abelian theory, we just need to find the oscillation frequency of an infrared mode (sufficiently infrared that we need not worry about nonrenormalizable corrections to its dispersion relation). The relation

$$
\frac{d}{d t} \epsilon^{i} A_{i}(k)=\epsilon^{i} E_{i}(k)
$$

holds in the abelian theory, for transverse fields and in temporal gauge. (Here $\epsilon^{i}$ is a unit vector satisfying $\epsilon^{i} k_{i}=0$. Henceforth we will write $\epsilon^{i} A_{i}$ simply as $A$.) Now $\left\langle A^{2}(k)\right\rangle$ receives the tadpole correction and is

$$
\left\langle A^{2}(k)\right\rangle=\frac{1}{k^{2} \beta_{L, \mathrm{imp}}},
$$

while the electric field appears quadratically in the Hamiltonian and will strictly obey equipartition,

$$
\left\langle E^{2}(k)\right\rangle=\frac{1}{\beta_{L}} .
$$

Hence the frequency squared of oscillation of this mode is $\omega^{2}=\left\langle E^{2}\right\rangle /\left\langle A^{2}\right\rangle=k^{2} \beta_{L \text {,imp }} / \beta_{L}$. The time scale is shifted by $\sqrt{\beta_{L} / \beta_{L \text {,imp }}}$ with respect to the length scale, and hence we should use $\sqrt{\beta_{L} \beta_{L, \mathrm{imp}}}$, not $\beta_{L, \text { imp }}$, when we relate the physical and lattice time scales.

This argument does not apply unmodified to the case of $\mathrm{SU}(2)$. For instance, the value of $\left\langle A_{i}^{2}(k)\right\rangle$ on the lattice depends on the renormalization of the $A$ field, which is gauge fixing dependent, and the condition $d A / d t=E$ is not true in temporal gauge on the lattice. However, these corrections do not arise at the level of the tadpole improvement approximation, because if they did they would be present already in the compact abelian theory; so we anticipate that they will be subdominant compared to the quite large $O(a)$ corrections present in $\beta_{L, \text { imp }}-\beta_{L}$, and that most $O(a)$ corrections will be absorbed by scaling between lattice and continuum time units using $\sqrt{\beta_{L} \beta_{L, \text { imp }}}$, as in the abelian theory. This is the approximation applied in the body of the paper.

\section{References}

[1] A. Sakharov, JETP Lett. 6 (1967) 24.

[2] G. t'Hooft, Phys. Rev. Lett. 37 (1976) 8. 
[3] V. Kuzmin, V. Rubakov, and M. Shaposhnikov, Phys. Lett. D 30 (1985) 36.

[4] F. Klinkhamer and N. Manton, Phys. Rev. D 30 (1984) 2212.

[5] P. Arnold and L. McLerran, Phys. Rev. D 36 (1987) 581.

[6] D. Grigorev and V. Rubakov, Nucl. Phys. B 299 (1988) 248.

[7] J. Ambjørn, M. Laursen, and M. Shaposhnikov, Nucl. Phys. B 316 (1989) , 483.

[8] D. Bodeker, L. McLerran, and A. Smilga, Phys. Rev. D 52 (1995) 4675.

[9] J. Ambjørn and A. Krasnitz, Phys. Lett. B 362 (1995) 97.

[10] W. Tang and J. Smit, Nucl. Phys. B 482 (1996) 265.

[11] G. D. Moore and N. Turok, Phys. Rev. D 55 (1997) 6538.

[12] D. Bodeker, Phys. Rev. D 53 (1996) 662.

[13] J. Ambjørn, T. Askgaard, H. Porter, and M. Shaposhnikov, Nucl. Phys. B 353 (1991) 346.

[14] G. D. Moore, Nucl. Phys. B 480 (1996) 657.

[15] G. D. Moore, Nucl. Phys. B 480 (1996) 689.

[16] P. Arnold, D. Son, and L. Yaffe, Phys. Rev. D 55 (1997) 6264.

[17] J. Ambjørn and A. Krasnitz, NBI-HE-97-18, hep-ph/9705380.

[18] S. Khlebnikov and M. Shaposhnikov, Nucl. Phys. B308 (1988) 885.

[19] V. Rubakov and M. Shaposhnikov, Phys. Usp. 39 (1996) 461-502, (Usp. Fiz. Nauk 166 (1996) 493-537.).

[20] P. Huet and D. Son, Phys. Lett. B393 (1997) 94.

[21] C. R. Hu and B. Müller, DUKE-TH-96-133, hep-ph/9611292.

[22] P. Woit, Phys. Rev. Lett. 51 (1983) 638; Nucl. Phys. B 262 (1985) 284.

[23] N. Turok, Phys. Rev. Lett. 63 (1989) 2625.

[24] V. N. Gribov, Nucl. Phys. B 139 (1978) 1.

[25] J. Mandula and M. Ogilvie, Phys. Lett B 185 (1987) 127.

[26] G. D. Moore, Nucl. Phys. B 493 (1997) 439.

[27] K. Farakos, K. Kajantie, K. Rummukainen, and M. Shaposhnikov, Nucl. Phys. B 442 (1995) 317.

[28] P. Arnold, Phys. Rev. D 55 (1997) 7781.

[29] G. P. Lepage and P. Mackenzie, Phys. Rev. D 48 (1993) 2250. 


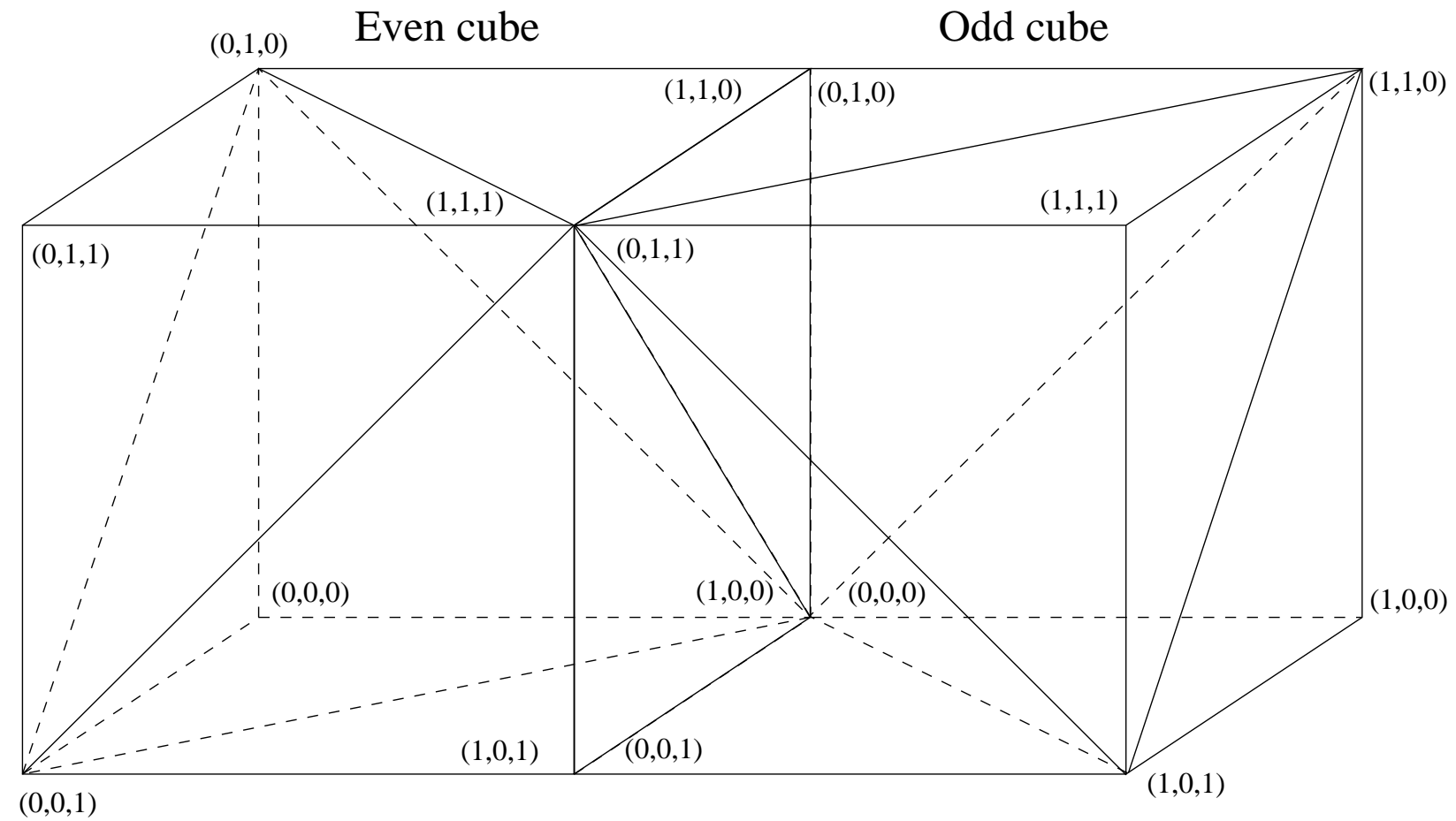

Vertices of tetrahedra, in positively oriented order: even cube

Vertices of tetrahedra, in positively

$(0,0,0),(1,0,0),(0,1,0),(0,0,1)$ oriented order: odd cube

$(1,0,0),(0,0,1),(1,0,1),(1,1,1)$

$(1,0,0),(1,1,0),(0,0,0),(1,0,1)$

$(0,1,0),(1,0,0),(1,1,0),(1,1,1)$

$(0,1,0),(1,1,0),(0,1,1),(0,0,0)$

$(0,0,1),(0,1,0),(0,1,1),(1,1,1)$

$(0,0,1),(1,0,1),(0,0,0),(0,1,1)$

$(1,0,0),(0,1,0),(0,0,1),(1,1,1)$

$(1,1,1),(1,1,0),(1,0,1),(0,1,1)$

$(0,0,0),(1,1,0),(0,1,1),(1,0,1)$

Figure 1: Triangulation into tetrahedra of two neighboring unit cubes, with the verticies of the tetrahedra, correctly ordered, listed below them. 


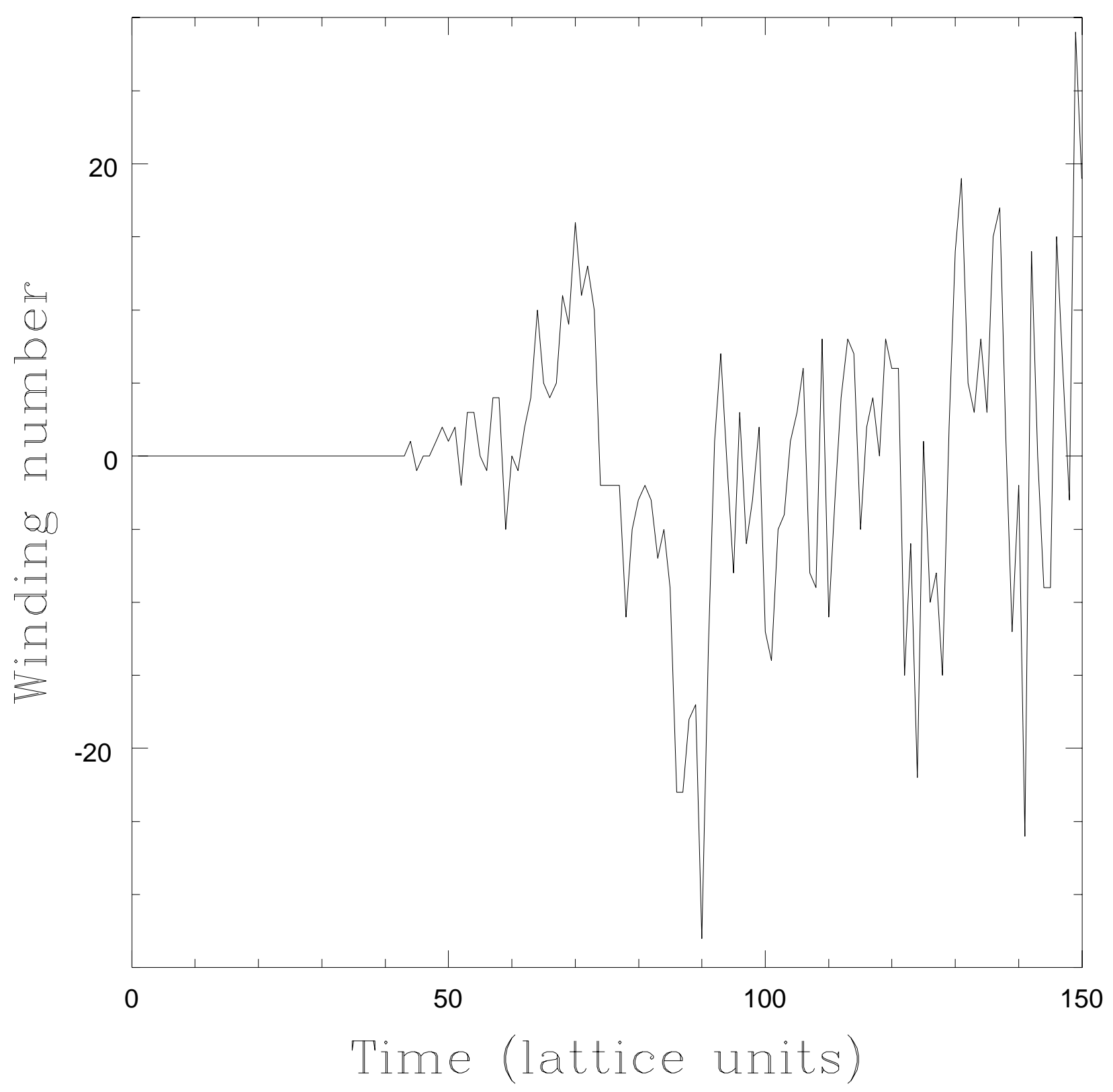

Figure 2: Evolution of winding number in the broken phase of Yang-Mills Higgs theory, starting with smooth connections and remaining in temporal gauge without ever performing small gauge changes to keep the connections smooth. The connections gradually become less smooth, so after a while the winding number begins to oscillate wildly. 


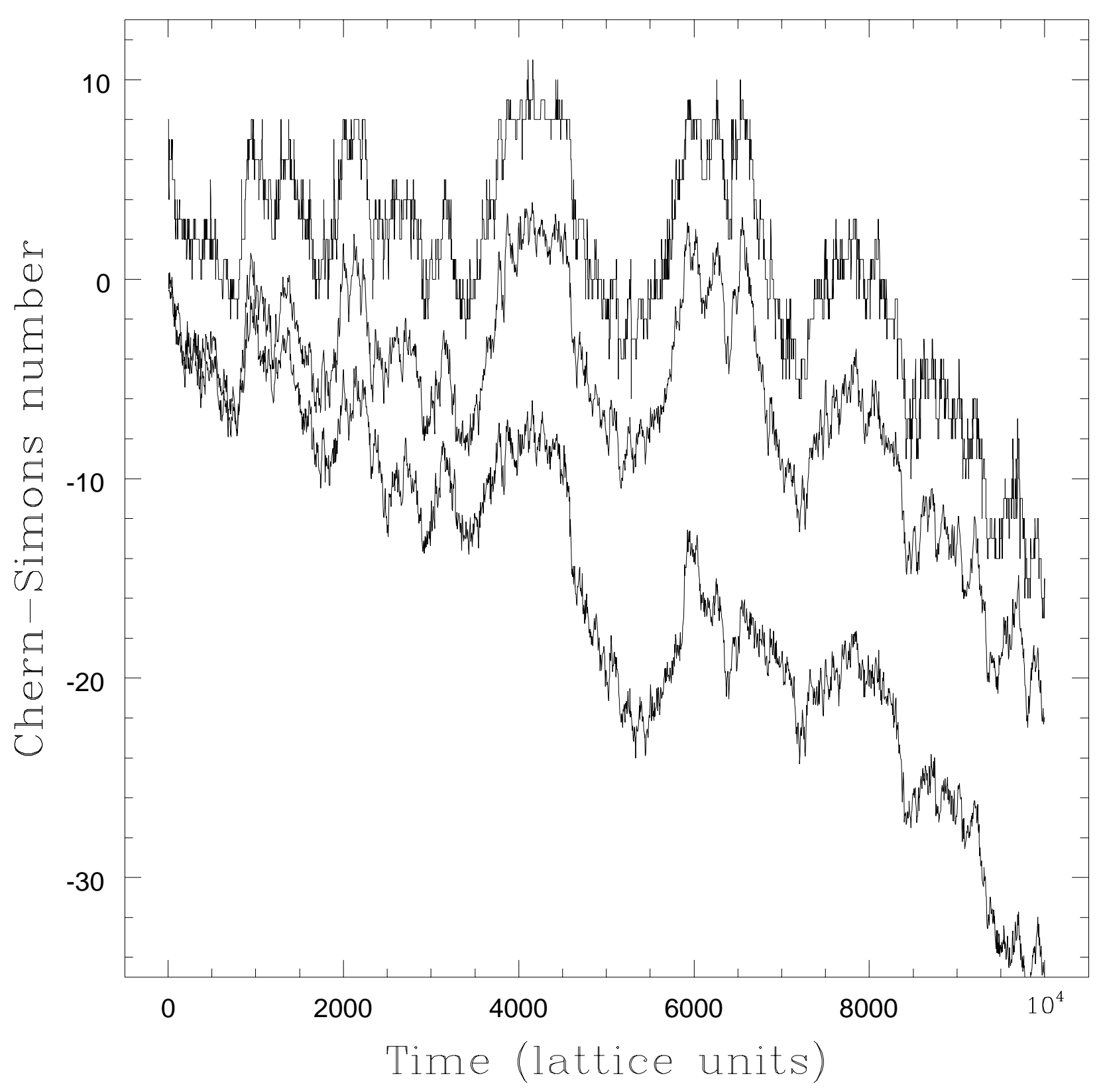

Figure 3: Chern-Simons number, tracked three ways, as a function of time, each for the same Hamiltonian evolution. The stairstep curve (top) is the winding number definition, the curve which tracks it closely is the cooled field definition, and the curve which drifts away from them is the old definition. The winding number definition has been shifted for clarity, otherwise it would land almost exactly on the cooled field definition. These two are very highly correlated, and the "old" definition is quite correlated with them but contains in addition some extra diffusive signal. 


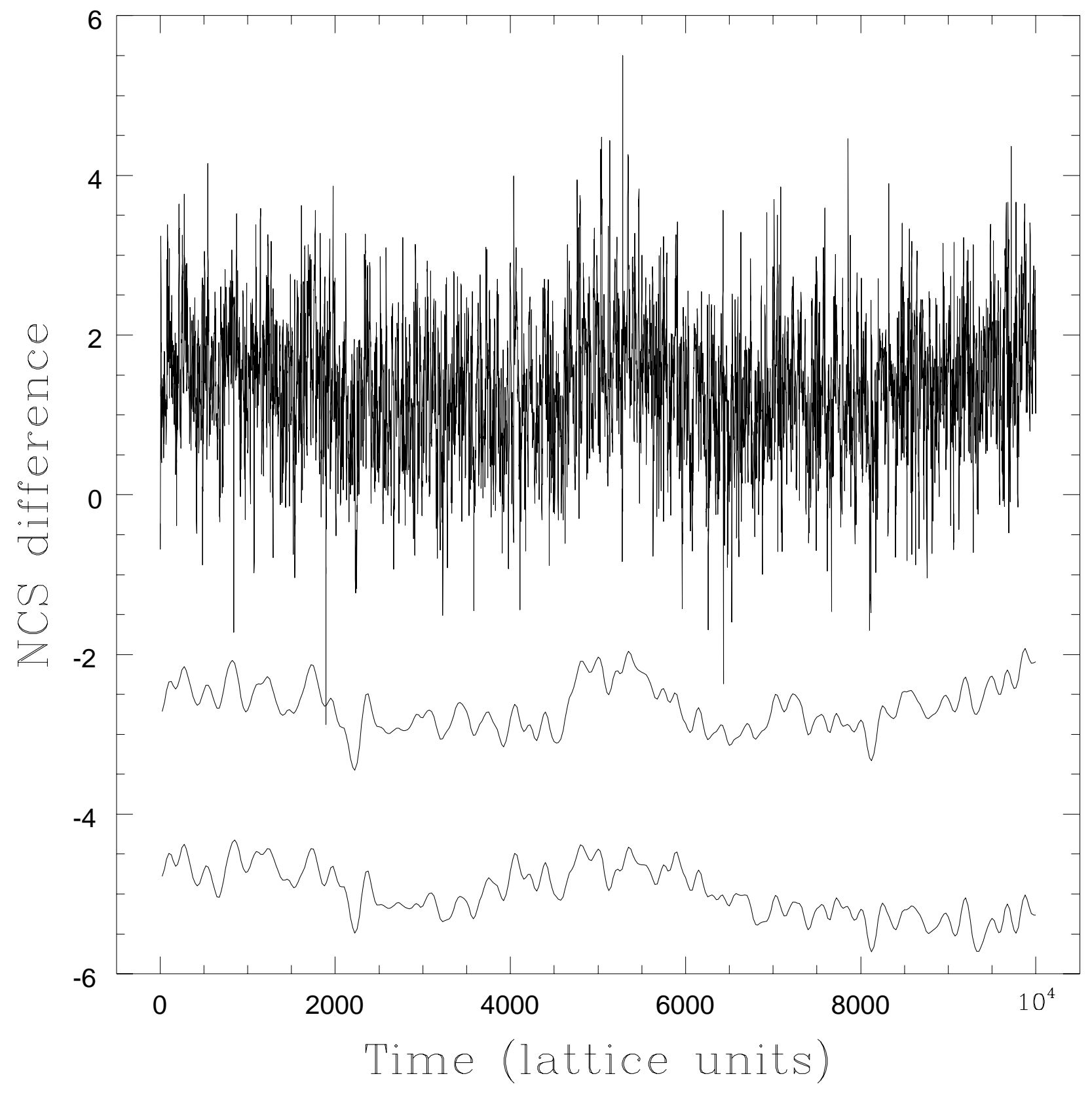

Figure 4: The difference between the winding number definition of $N_{C S}$ and the cooled field definition (top curve), with moving average (shifted for clarity, middle curve), and the moving average after correlations with the cooled field definition have been removed (bottom curve). The correlations mean that the cooled field receives a multiplicative correction with respect to the winding number (topological) definition, probably due to nonrenormalizable operators. 


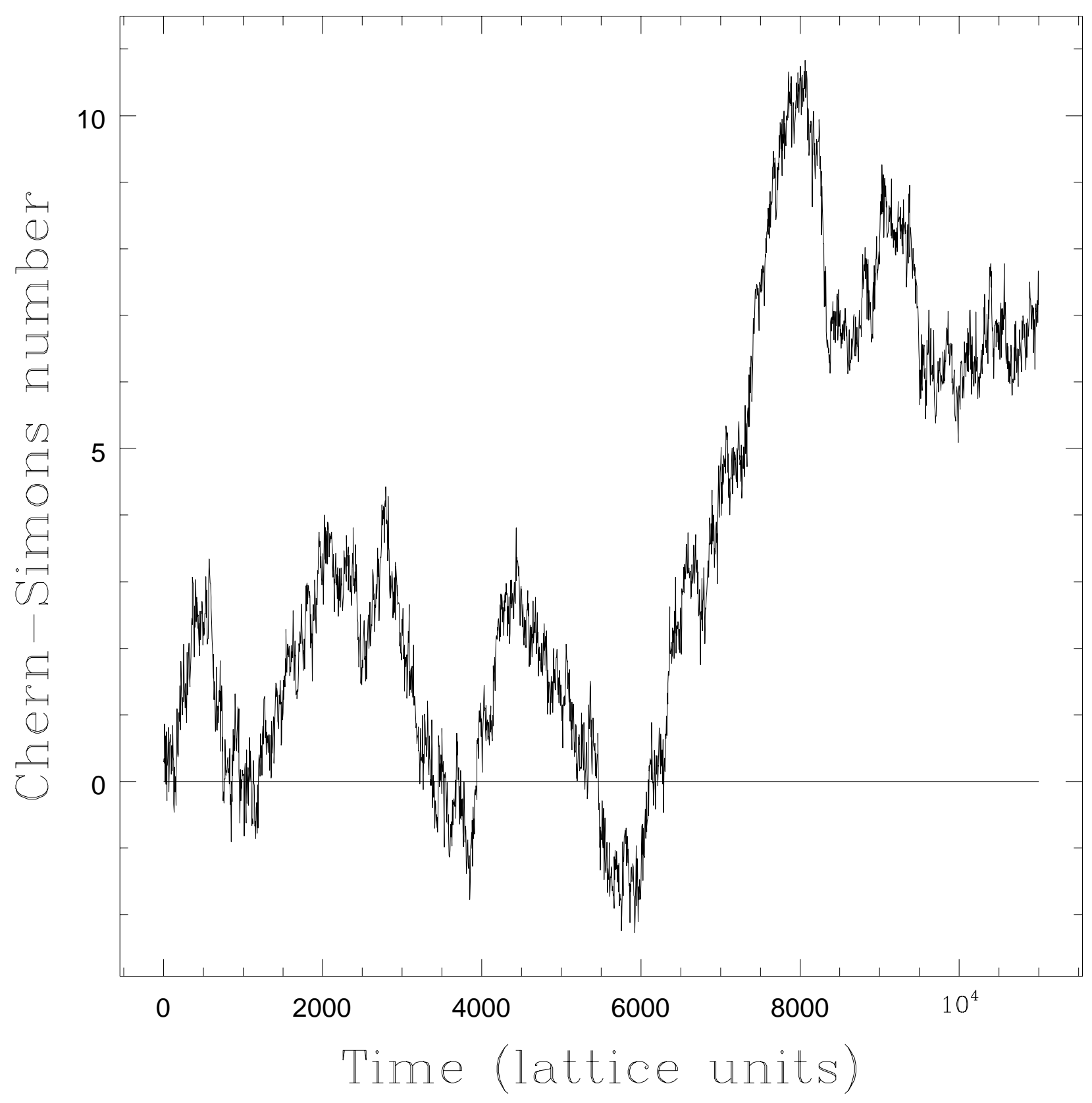

Figure 5: winding number (constant line) and old definition of $N_{C S}$ for a Hamiltonian trajectory in the broken electroweak phase at the phase transition temperature. There are no winding number changes in the trajectory; the diffusion of $N_{C S}$ under the old definition is a lattice artifact. 


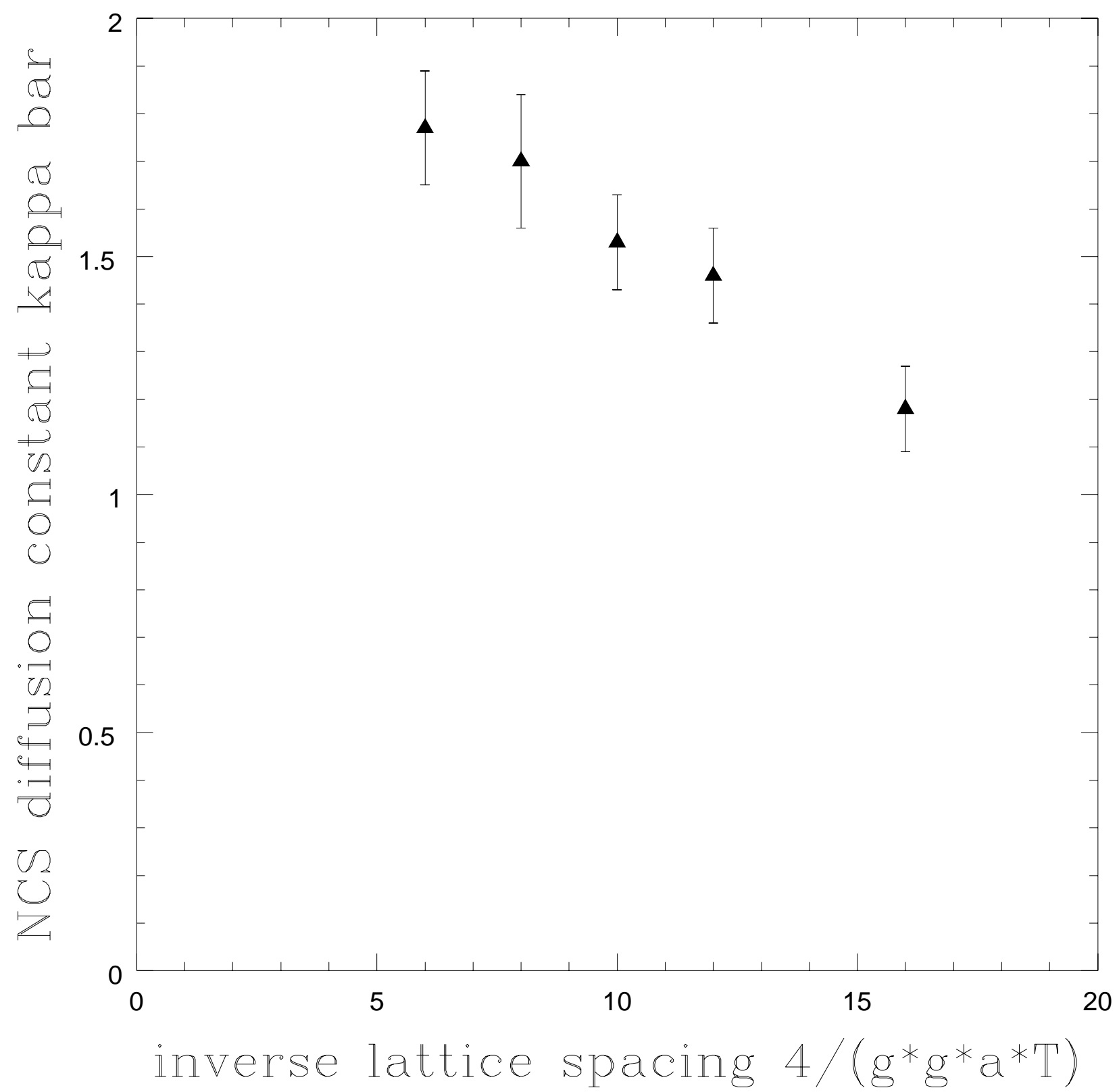

Figure 6: Results for $\bar{\kappa}$ as a function of lattice spacing in Yang-Mills theory. The dependence on $\beta_{L}$ is strong, but not as strong as $\beta_{L}^{-1}$. 


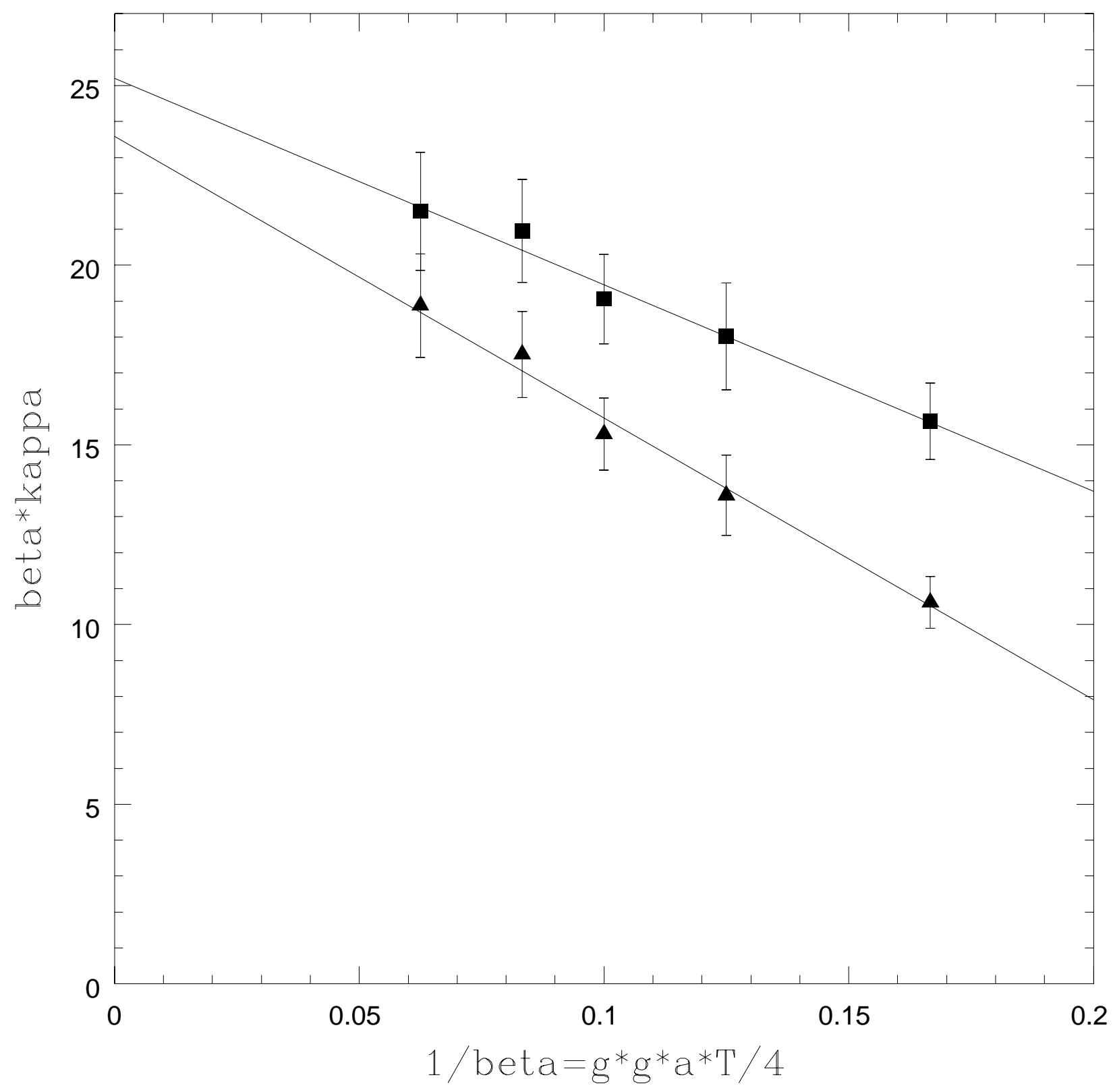

Figure 7: $\bar{\kappa} \times \beta_{L}$ (triangles) and $\kappa \times \beta_{L}$ (squares) plotted against $\beta_{L}^{-1}$. The arguments of Arnold, Son, and Yaffe imply a good small $\beta_{L}^{-1}$ (small a) limit. We display a linear fit; the extrapolation seems plausible but the fit should not be overinterpreted. 